\title{
Downregulation of c-Myc is involved in TLR3-mediated tumor death of neuroblastoma xenografts
}

\author{
Li-Ling Lin ${ }^{1,2,6}$, Chao-Cheng Huang ${ }^{3,4,6}$, Chia-Ling Wu' ${ }^{1}$ Min-Tsui Wu', Wen-Ming Hsu ${ }^{5}$ and Jiin-Haur Chuang ${ }^{1}$
}

Neuroblastoma (NB) is the deadliest pediatric solid tumor due to its pleomorphic molecular characteristics. In the innate immune system, toll-like receptor 3 (TLR3) recognizes viral double-stranded RNAs to initiate immune signaling. Positive TLR3 expression indicates a favorable prognosis in NB patients, and is associated with MYCN-non-amplified. However, TLR3-mediated innate immune responses remain elusive in NB. In this study, we attempted to dissect the molecular mechanism underlying TLR3-agonist polyinosinic-polycytidylic acid [poly(l:C)] treatment in NB in vivo. We established NB xenograft models in non-obese diabetic/severe combined immunodeficiency (NOD/SCID) mice with MYCN-amplified SK-N-DZ (DZ) cells or MYCN-non-amplified SK-N-AS (AS) cells. Poly(I:C) treatment led to significant tumor regression in AS xenografts, but not in DZ xenografts. Through immunohistochemical analysis, significant suppression of tumor proliferation, downregulation of c-Myc expression, and upregulation of TLR3 expression were found in the treatment group. Poly $(\mathrm{I}: \mathrm{C})$ inducing activation of TLR3/IRF3-mediated innate immunity associated with downregulation of c-Myc can be found in MYCN-non-amplified SK-N-AS cells, but not in MYCN-amplified BE(2)-M17 cells. Knockdown of TLR3 disturbed poly(I:C)-induced suppression of c-Myc and upregulation of p-IRF3 in AS cells. Furthermore, poly(l:C) treatment upregulated active NF-KB, mitochondrial antioxidant manganese superoxide dismutase and 8-hydroxydeoxyguanosine, which works with reactive oxygen species (ROS) generation and DNA damage. Upregulation of active caspase 3 and cleaved poly [ADP-ribose] polymerase 1 were found in poly(l:C)-treated AS xenografts, which indicates the induction of apoptosis. Thus, our results suggest that c-Myc overexpression may increase sensitivity to poly(l:C)-induced tumor growth arrest and ROS-mediated apoptosis in NB. This study demonstrates that c-Myc protein expression has an important role in TLR3-induced innate immune responses, providing future treatment recommendations.

Laboratory Investigation (2016) 96, 719-730; doi:10.1038/labinvest.2016.57; published online 16 May 2016

Neuroblastoma (NB) is a common solid tumor of the peripheral nervous system, arising from the adrenal medulla and accounting for $15 \%$ of childhood cancer mortality. ${ }^{1,2}$ More than $90 \%$ of NB patients are diagnosed in children under 10 years old and accompanied with pleomorphic molecular characteristics, such as abnormalities of chromosome $1 \mathrm{p}, 11 \mathrm{q}$ or $17 \mathrm{q}$ as well as MYCN amplification. ${ }^{3-5}$ To define risk category, several crucial clinical characters, including MYCN (v-myc avian myelocytomatosis viral oncogene neuroblastoma derived homolog) status, histopathology, patient's age and tumor stage (International Neuroblastoma Staging System, INSS) at diagnosis, have been used to determine the optimal therapeutic strategy for NB patients. ${ }^{4}$ In the past two decades, the 5 -year survival rates in patients with low- and intermediate-risk NB has been more than $90 \% .{ }^{6,7}$ Instead, the overall survival rate in patients with refractory high-risk NB ( $45 \%$ of total NBs) is still only $30-40 \%$ due to clinical heterogeneity and disease recurrence, despite intensive treatment with surgery, chemotherapy and radiation therapy. ${ }^{1}$ Nevertheless, neoadjuvant or adjuvant chemotherapy is still the mainstream for the treatment of NB. Discovery of biomarkers for more effective treatment of NBs with distinct molecular backgrounds, particularly for high-risk $\mathrm{NB}$, remains urgent in the era of precision medicine.

Myc oncogenes, including MYC and MYCN, have been found to be associated with aggressive clinical behavior in NB. ${ }^{8,9}$ MYCN amplification, occurring in about $25 \%$ of NB, is known to be a poor prognostic factor. However, the incidence

\footnotetext{
'Department of Pediatric Surgery, Kaohsiung Chang Gung Memorial Hospital, Chang Gung University College of Medicine, Kaohsiung, Taiwan; ${ }^{2}$ Department of Medical Research, Kaohsiung Chang Gung Memorial Hospital, Chang Gung University College of Medicine, Kaohsiung, Taiwan; ${ }^{3}$ Biobank and Tissue Bank, Kaohsiung Chang Gung Memorial Hospital, Kaohsiung, Taiwan; ${ }^{4}$ Department of Pathology, Kaohsiung Chang Gung Memorial Hospital and Chang Gung University College of Medicine, Kaohsiung, Taiwan and ${ }^{5}$ Department of Surgery, National Taiwan University Hospital and National Taiwan University College of Medicine, Taipei, Taiwan

Correspondence: Dr J-H Chuang, MD, Department of Pediatric Surgery, Kaohsiung Chang Gung Memorial Hospital, 123 Ta-Pei Road, Niao-Song District, Kaohsiung 833, Taiwan. E-mail: jhchuang@cgmh.org.tw

${ }^{6}$ These authors contributed equally to this work.
}

Received 4 January 2016; revised 29 March 2016; accepted 31 March 2016 
of MYCN amplification may also be affected by other factors, such as tumor cell ploidy, localized disease, and stage of NB. ${ }^{9-11}$ Recent studies showed that c-Myc protein expression acts as a poor prognostic feature associated with more aggressive clinical behaviors than MYCN amplification alone or N-Myc protein expression. ${ }^{8,12,13}$ Through triggering epigenetic changes, $M Y C N$-non-amplified NB cells could be transformed to aggressive, stem cell-like cells. ${ }^{14}$ In up to $70 \%$ of human cancers, c-Myc protein expression is deregulated and considered as an attractive target for cancer therapy. ${ }^{15}$ Its transcriptional targets may promote cell cycle progression, tumor cell growth, and cell differentiation, and regulate cellular metabolism and cell adhesion as well as apoptosis. ${ }^{16} \mathrm{In}$ proliferating cells, overexpression of c-Myc coupled with antiapoptotic mutation facilitates tumor invasion and progression. ${ }^{16}$ Downregulation of c-Myc in cancer cells can lead oncogene addiction to be elicited followed by cell growth arrest, senescence, promoting apoptosis, and tumor regression. ${ }^{17}$

Recently, immunotherapy has become the limelight for the treatment of children with aggressive and high-risk NB to improve survival and reducing relapse rate. ${ }^{18,19}$ Our previous studies disclosed that a high level of Toll-like receptor 3 (TLR3) is associated with a favorable prognosis in NB patients, and different levels of TLR3 expression are shown in NB cell lines. ${ }^{20,21}$ TLR3, which is localized in endosomes or on cell surfaces in conventional dendritic cells, serves as a sensor of viral infection to recognize double-stranded RNA and triggers antiviral signal transduction in innate immunity. ${ }^{22}$ Being an agent related to TLR3 agonist-based immunotherapy, polyinosinic-polycytidylic acid [poly(I:C)] has been used as a cancer vaccine adjuvant in several types of cancers, such as prostate cancer, lymphoma, lung cancer, melanoma, and hepatocellular carcinoma. ${ }^{23,24}$ In human renal cell carcinoma cells, poly(I:C) treatment can downregulate c-Myc expression and facilitate reactive oxygen species (ROS) generation to induce DNA damage, cell growth arrest, and apoptosis via innate adjuvant receptors and the $2-5 \mathrm{~A}$ system. ${ }^{25}$ In NB, we have found that poly(I:C) may induce tumor cells apoptosis, preferentially through the mitochondrial pathway. ${ }^{21,26}$ Several TLR3 agonist-induced signals in suppression of NB cells have been shown, including activation of protein kinase $\mathrm{R}$, interferon regulatory factor 3 (IRF3), mitochondrial antioxidant manganese superoxide dismutase (MnSOD), and caspase $3 .^{21,26}$ In addition, we found that another viral RNA sensor, melanoma differentiation-associated antigen-5 (MDA5), can complement TLR3 to improve poly(I:C) suppression of NB. ${ }^{27}$ These studies demonstrate that poly $(\mathrm{I}: \mathrm{C})$ is a potential therapeutic strategy for NB, and may be applied in clinical treatment in the future. However, the effects and underlying mechanism of poly(I:C) in animal model of NB remains elusive.

The purpose of the present study was to investigate whether poly(I:C) treatment can induce tumor death in NB xenografts. The non-obese diabetic/severe combined immunodeficiency
(NOD/SCID) mice model of NB cells has been widely used to investigate pathogenesis and develop therapeutic strategies in clinical practice. ${ }^{28}$ Here we subcutaneously injected NOD/ SCID mice with $M Y C N$-non-amplified (SK-N-AS, AS) or $M Y C N$-amplified (SK-N-DZ, DZ) NB cells to elucidate the effects of poly(I:C) treatment for $\mathrm{NB}$ in vivo. Our results indicated that poly(I:C) significantly shrank tumors in AS xenograft mice, but not in DZ xenograft mice. c-Myc protein expression was significantly downregulated by poly(I:C) treatment in AS xenografts. Silencing TLR3 attenuated the suppression effect on c-Myc protein expression in poly(I:C)treated AS cells. The activation of IRF3, NF- $\mathrm{KB}, \mathrm{MnSOD}$, caspase 3, and poly (ADP-ribose) polymerase 1 (PARP-1) was triggered by poly(I:C). Finally, downregulation of c-Myc might be involved in TLR3-induced tumor growth arrest and apoptosis of NB xenografts through DNA damage and generation of ROS.

\section{MATERIALS AND METHODS Cell Lines and Culture Conditions}

Human NB cell lines, SK-N-AS (CRL-2137), SK-N-DZ (CRL-2149), and BE(2)-M17 (CRL-2267), were purchased from the American Type Culture Collection (ATCC, Manassas, VA, USA). SK-N-AS and SK-N-DZ cell lines were cultured in Dulbecco's modified Eagle's medium (DMEM) adjusted to contain $2 \mathrm{mM}$ L-glutamine. BE(2)-M17 cells were cultured in Minimum Essential Media (MEM)/F-12 (1:1) medium adjusted to contain $1 \mathrm{mM}$ sodium pyruvate. All culture media were supplemented with $10 \%(\mathrm{v} / \mathrm{v})$ heatinactivated fetal bovine serum (FBS), $10 \mathrm{mM}$ nonessential amino acids, and antibiotic-antimycotic. Cells were cultured at $37^{\circ} \mathrm{C}$ in an incubator with controlled humidified atmosphere containing $5 \% \mathrm{CO}_{2}$. All cell culture reagents were purchased from Invitrogen (Carlsbad, CA, USA).

\section{Human Tumor Xenografts}

Male NOD/SCID mice, 4 weeks of age, were purchased from BioLASCO Taiwan Co. (Ilan, Taiwan). All animal procedures were approved by the Animal Ethics Committee of the Kaohsiung Chang Gung Memorial Hospital. After 1 week of adaptation, SK-N-DZ cells $\left(1 \times 10^{7}\right.$ cells $)$ or SK-N-AS cells $\left(5 \times 10^{6}\right.$ cells $)$ were injected subcutaneously into the right flank of NOD/SCID mice. Tumor size was documented with caliper measurement every 3 days. When the tumors grew to $\sim 4-6 \mathrm{~mm}$ in diameter by 1 week after implantation, the mice received an intraperitoneal injection of normal saline (control group) or $10 \mathrm{mg} / \mathrm{kg}$ of poly(I:C) (Invivogen, San Diego, CA, USA) (treatment group) twice a week. The mice were killed on the second day after the last treatment, which was day 17 or day 27 after injection. Thereafter, the tumor size and weight were measured as previously described. ${ }^{29}$ A part of each tumor was then fixed in formalin overnight and embedded in a paraffin block for immunohistochemical staining (IHC). A total of 47 mice were available at the completion of the xenograft study (AS xenografts: on day 17, 
control group $n=6$ and treatment group $n=7$; on day 27, control group $n=6$ and treatment group $n=6 . \mathrm{DZ}$ xenografts: on day 17, control group $n=3$ and treatment group $n=5$; on day 27 , control group $n=7$ and treatment group $n=7)$.

\section{RNAi and Transfection}

Human TLR3-specific stealth siRNA (siTLR3) and negative control (scrambled) siRNA were purchased from Invitrogen (Carlsbad, CA, USA). The siTLR3 sequence was 5'-CCT GAGCTGTCAAGCCACTACCTTT- $3^{\prime}$, and the scrambled sequence was 5'-CCTGTCGAACTACCGCATCCAGTTT-3'. SK-N-AS cells and BE(2)-M17 cells were transfected with siRNA ( $25 \mathrm{nM}$ ) using Lipofectamine RNAiMAX (Invitrogen) according to the manufacturer's instructions.

\section{Western Blot Analysis}

After siRNA transfection for $24 \mathrm{~h}, \mathrm{SK}-\mathrm{N}-\mathrm{AS}$ cells and BE(2)M17 cells were treated with $50 \mu \mathrm{g} / \mathrm{ml}$ poly(I:C) for another $24 \mathrm{~h}$, then the cell lysates were prepared. Cells were lysed with protein extraction solution containing proteases inhibitors (iNtRON Biotechnology, Sungnam, Kyungki-Do, Korea). Samples were separated by $8 \%$ SDS-PAGE and then transferred onto nitrocellulose membrane. The membrane was blocked for $1 \mathrm{~h}$ at room temperature in $5 \%$ nonfat milk and incubated overnight at $4{ }^{\circ} \mathrm{C}$ with the following primary antibodies: anti-p-IRF3 (phospho Ser386, 1:1000, Epitomics, Burlingame, CA, USA), anti-IRF3 (1:1000, Epitomic), anti-cMyc antibody (1:1000, Cell Signaling Technology, Danvers, MA, USA) and anti-N-Myc antibody (1:1000, Cell Signaling Technology). Membranes were then incubated with HRPconjugated anti-mouse (1:10 000, Jackson ImmunoResearch, Newmarket, Suffolk, UK) and anti-rabbit (1:5000, Jackson ImmunoResearch) secondary antibodies for $1 \mathrm{~h}$ at room temperature. $\beta$-actin (1:10 000, Millipole, Billerica, MA, USA) antibody was used as an internal control. Protein-antibody complexes were visualized with an enhanced chemiluminescence (ECL) detection kit (Amersham Pharmacia Biotech, Uppsala, Sweden) and visualized on X-ray films. Signals were quantified by densitometric analysis.

\section{Immunohistochemistry}

For immunohistochemical staining (IHC), paraffin sections $(2 \mu \mathrm{m})$ were cut from formalin-fixed-paraffin-embedded block of the xenograft tissue. After incubation overnight at $55^{\circ} \mathrm{C}$, the sections were deparaffinized in xylene and hydrated through descending grades of ethanol to deionized water. Antigen retrieval was performed by boiling the sections in citrate buffer $(10 \mathrm{mM}, \mathrm{pH}$ 6, Thermo Fisher Scientific, Waltham, MA, USA) for $30 \mathrm{~min}$. Further, endogenous peroxidase activity was blocked with 3\% hydrogen peroxide solution (Thermo Fisher Scientific) for $10 \mathrm{~min}$. The sections were then incubated with primary antibody: anti-c-Myc antibody (1:50, Abcam, Cambridge, UK) or anti-PARP-1 (cleaved Asp214) antibody (1:25; Cell Signaling Technology) overnight at $4{ }^{\circ} \mathrm{C}$. Several other primary antibodies were also used with incubation for $1 \mathrm{~h}$ at room temperature: anti-Ki-67 antibody (1:200; Abcam), anti-GAP43 (phospho S41) antibody (1:100; Abcam), anti-TLR3 antibody (1:100; Abcam), anti-caspase 3 antibody (1:100; Abcam), anti-NF-кB p65 (phospho Ser536) antibody (1:25; Santa Cruz Biotechnology, Santa Cruz, CA, USA), anti-SOD-2 (B-1) (MnSOD) antibody (1:50; Santa Cruz Biotechnology), anti-8-OHdG antibody (1:100; Japan Institute for the Control of Aging, Nikken SEIL Co.), and anti-IRF3 (phospho Ser396) antibody (1:50; Biorbyt, Cambridge, Cambridgeshire, UK). Next, tissue sections were incubated with Primary Antibody Amplifer Quanto (Ultravision Quanto, Thermo Fisher Scientific) for $10 \mathrm{~min}$, and then HRP Polymer Quanto (Ultravision Quanto detection system, Thermo Fisher Scientific) for $10 \mathrm{~min}$. Finally, the sections were rinsed with PBS followed by visualization with ImmPACT diaminobenzidine (DAB) peroxidase substrate (Vector Laboratories, Burlingame, CA, USA). After all, the sections were counterstained with Mayer's hematoxylin (ScyTek Laboratories, Logan, UT, USA) and mounted.

\section{Quantification of Immunohistochemical Staining}

To quantitatively analyze the expressions of Ki-67, p-GAP43, c-Myc, TLR3, p-IRF3, p-NF-кB p65, MnSOD, 8-OHdG, active caspase 3 , and cleaved PARP-1 by IHC, we randomly selected five high-power $(\times 400)$ fields for each section to evaluate each sample. The intensity of positive staining tumor cells was scored as: $0=$ none; $1=$ weak; $2=$ intermediate and $3=$ strong. The proportion of each intensity score was further scored as: $0=$ no positive cells; $1=0-20 \% ; 2=21-50 \%$; $3=51-80 \% ; 4=81-100 \% .^{30,31}$ Both scores were multiplied and summed to produce a final immunoreactivity score (IRS) ranged 0 to 12 .

\section{Statistical Analysis}

Statistical analyses were performed using SPSS software (version 12.0 for Windows; SPSS, Chicago, IL, USA). Data were expressed as mean \pm s.e.m., except western blot analysis, for which data were expressed as mean \pm s.d. The differences between control and treatment groups were tested by using a two-tailed Student's $t$-test. A $P$-value $<0.05$ was considered to be statistically significant.

\section{RESULTS \\ TLR3 Agonist-Poly(I:C) Significantly Suppresses the Tumor Growth in AS Xenografts, but not in DZ Xenografts}

To explore the effects of TLR3 agonist-poly(I:C) treatment in vivo, we established xenograft tumor models in NOD/SCID mice by subcutaneous injection of AS ( $M Y C N$-non-amplified) or DZ (MYCN-amplified) NB cells. After 1 week, NB xenografts were treated with $10 \mathrm{mg} / \mathrm{kg}$ of poly(I:C) (treatment group) or normal saline (control group). We measured the tumor size and weight of NB xenografts on day 17 and day 27 to monitor the effectiveness of poly $(\mathrm{I}: \mathrm{C})$ treatment (Figure 1 
and Supplementary Figure S1). Intriguingly, in AS xenografts on day 17, the tumor size of the treatment group was decreased (Figure 1b), with a significantly decreased tumor weight to $0.01 \pm 0.005 \mathrm{~g}$ compared with the control group $0.10 \pm 0.03 \mathrm{~g} \quad(P=0.023$, Figure 1c). Similarly, in
AS xenografts on day 27, poly(I:C) significantly reduced the tumor size to $203 \pm 106.95 \mathrm{~mm}^{3}(P=0.015$, Figure 1d) and the tumor weight to $0.44 \pm 0.12 \mathrm{~g}(P=0.03$, Figure 1e) compared with the control group $\left(1562 \pm 382.87 \mathrm{~mm}^{3}\right.$ and $2.26 \pm 0.50 \mathrm{~g})$. However, no significant changes between a

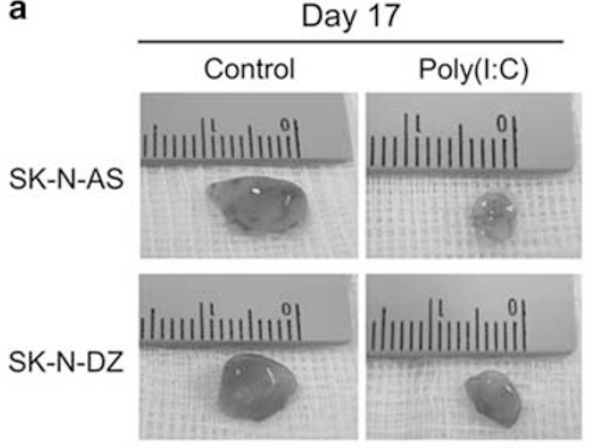

b

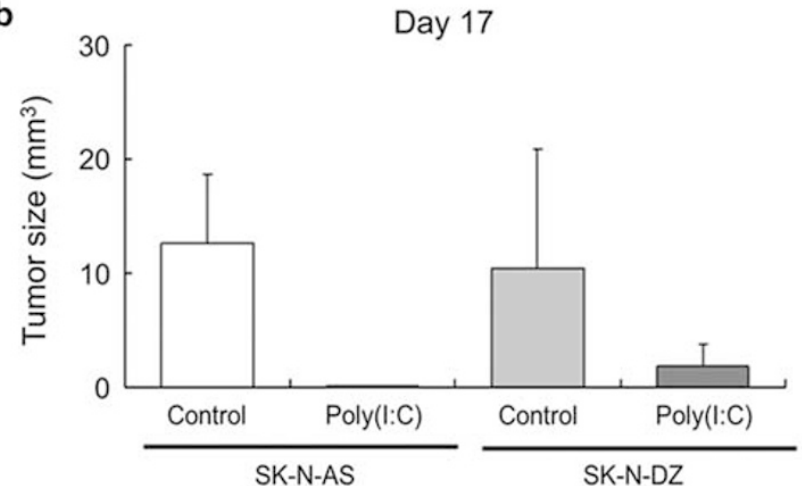

d

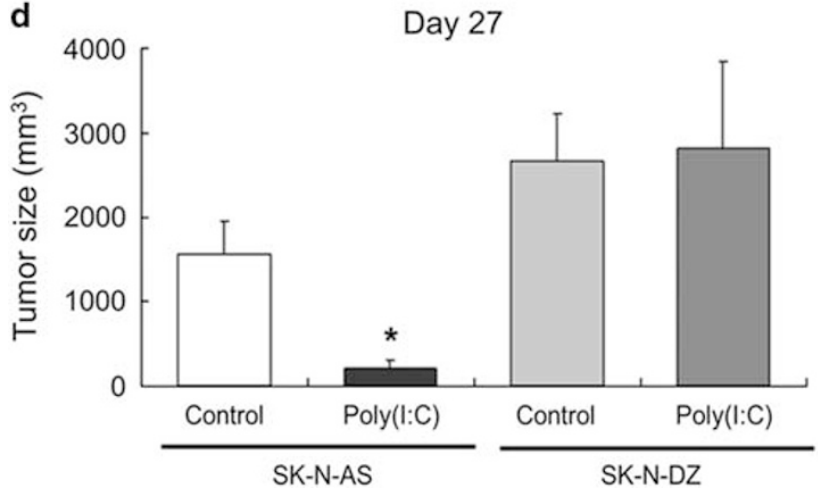

Day 27
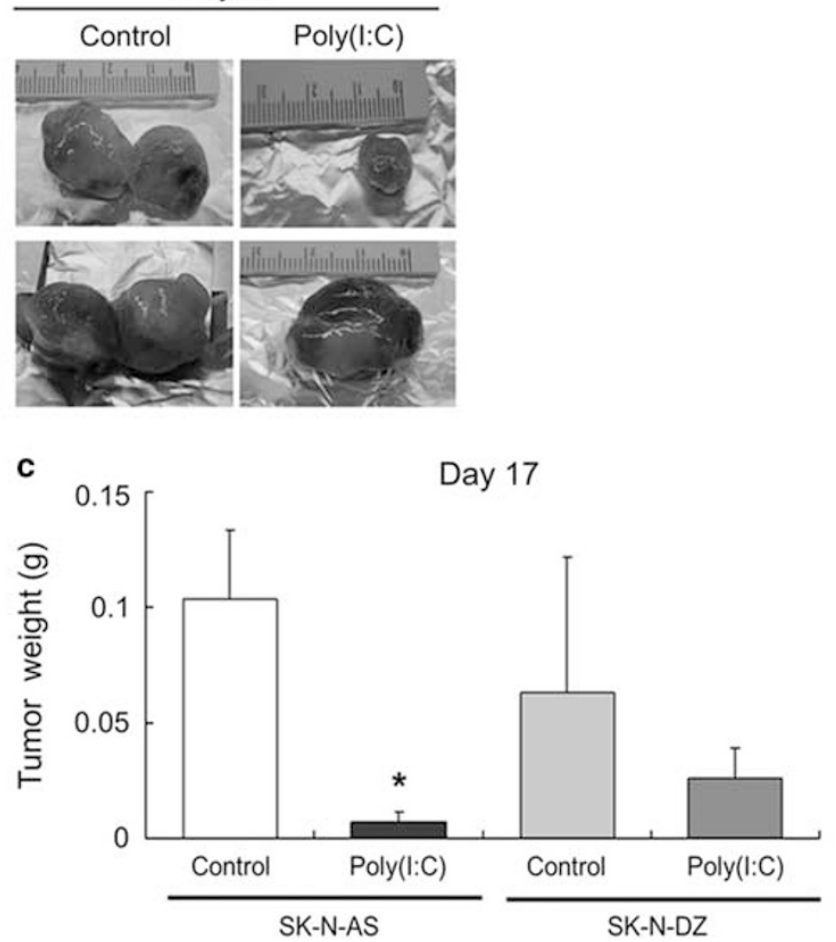

e

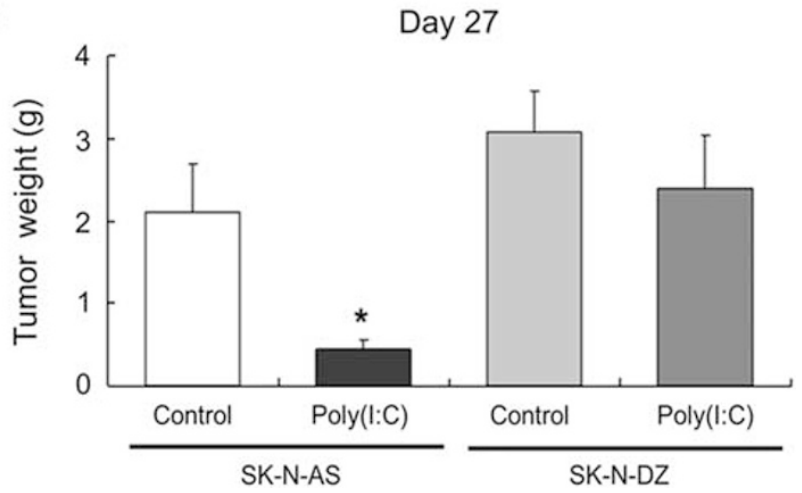

Figure 1 Reduction of tumor size and weight of SK-N-AS (AS) but not SK-N-DZ (DZ) xenografts in NOD/SCID mice with poly(l:C) treatment. AS cells $\left(5 \times 10^{6}\right)$ or DZ cells $\left(1 \times 10^{7}\right)$ were subcutaneously injected into NOD/SCID mice. (a) Representative AS or DZ xenografts on day 17 and day 27 are presented. (b and c) Tumor size and tumor weight of NB xenografts on day 17 were measured. AS xenografts: control group $n=6$, treatment group $n=7$. DZ xenografts: control group $n=3$, treatment group $n=5$. (d and e) Tumor size and tumor weight of NB xenografts on day 27 were measured. AS xenografts: control group $n=6$, treatment group $n=6$. DZ xenografts: control group $n=7$, treatment group $n=7$. Data are expressed as means \pm s.e.m. of mice in each group. Quantitative analysis of tumors was determined as described in Materials and Methods. ${ }^{*} P<0.05$ with the control group.

Figure 2 Decrease in Ki-67 and p-GAP43 immunohistochemical stainings in SK-N-AS xenografts with poly(I:C) treatment. (a) Representative images of immunohistochemical staining for Ki-67 and p-GAP43 performed on tissue sections from SK-N-AS xenografts with or without poly(I:C) treatment. (b) Quantitative analysis of Ki-67 by immunoreactivity score. (c) Quantitative analysis of p-GAP43 by immunoreactivity score. Data represent mean immunoreactivity score \pm s.e.m. for each group. Original magnification, $\times 400$. Scale bar, $25 \mu$ m. ${ }^{* * *} P<0.001$. 
a
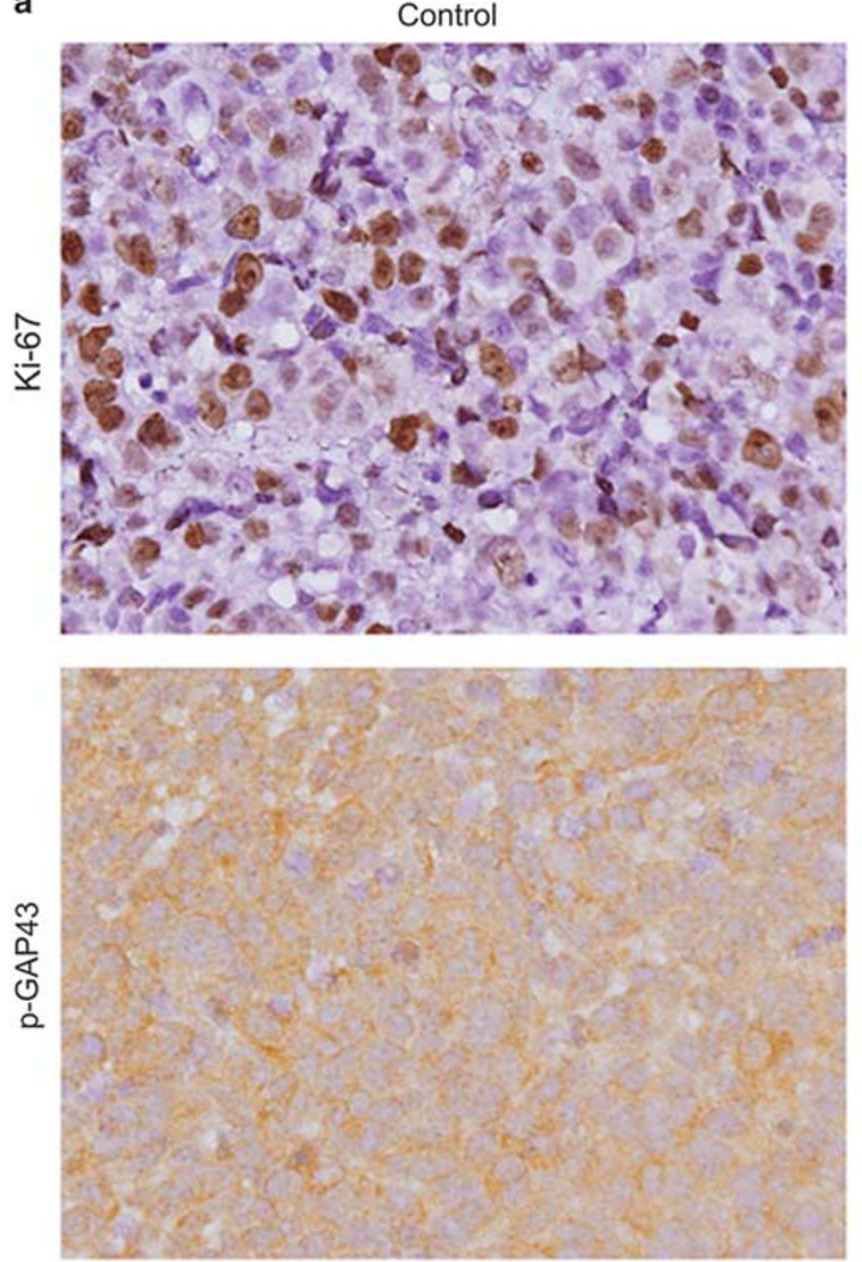

b

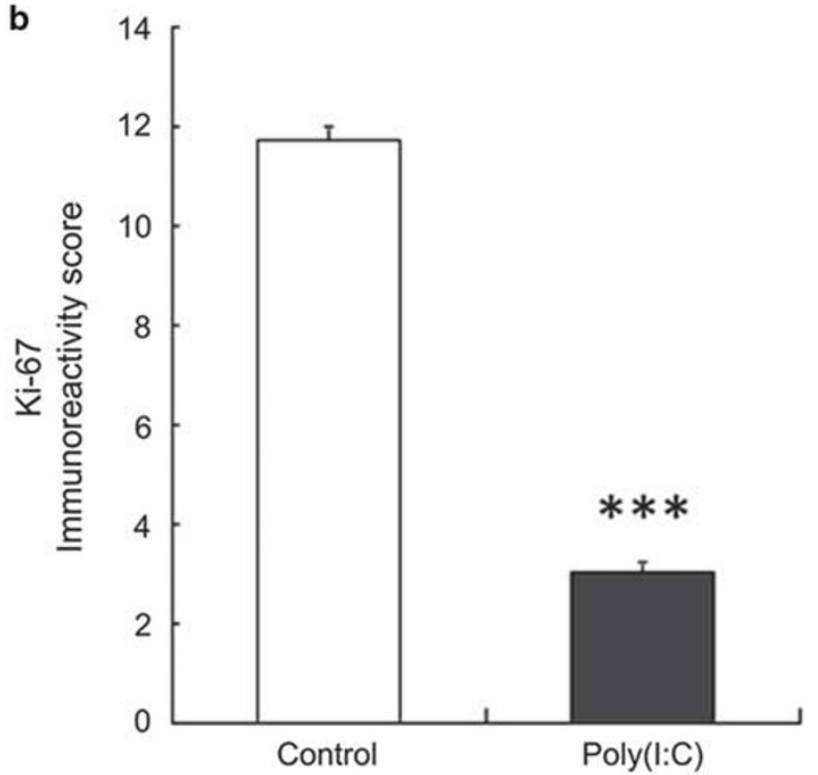

Poly(I:C)
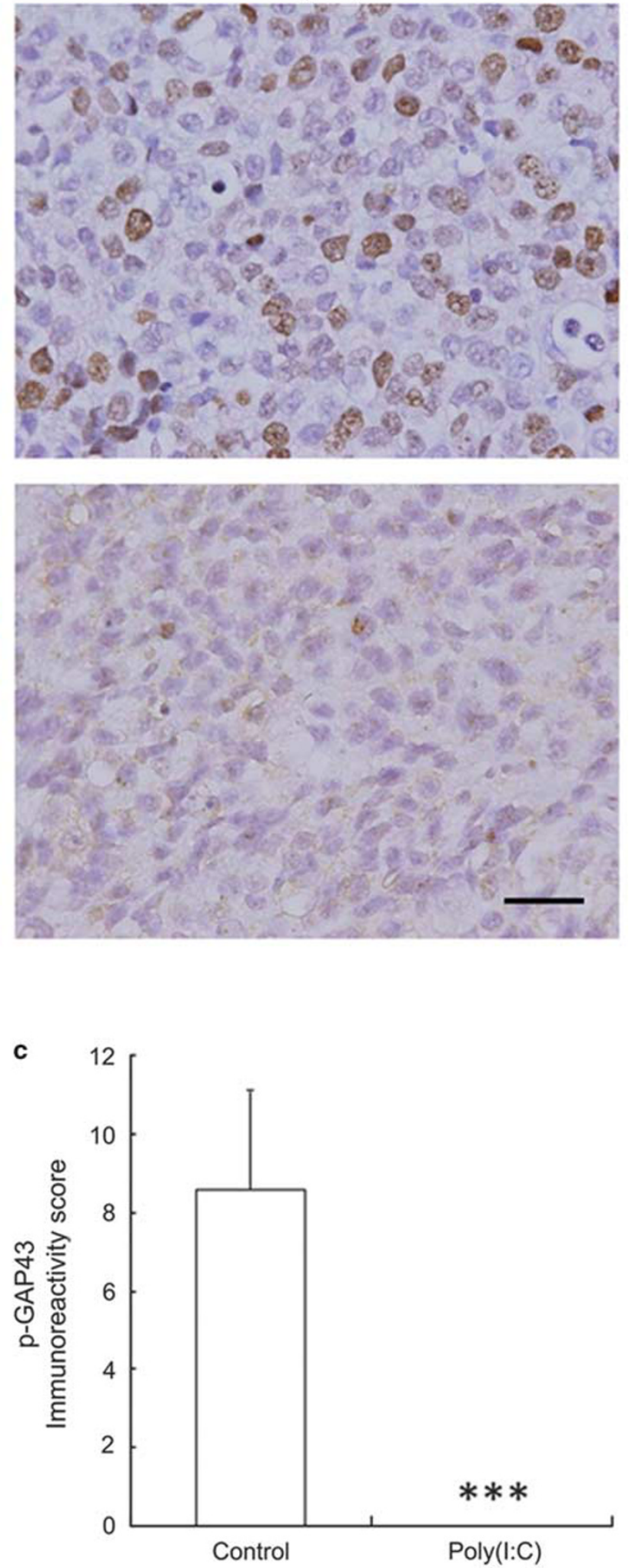
treatment group and control group were found in DZ xenografts on day 17 or day 27 , even if a small decrease was found in $\mathrm{DZ}$ xenografts on day 17 (Figures $1 \mathrm{~b}$ and c). In vitro experiments, we also found that poly(I:C) treatment can significantly suppress the cell growth in $M Y C N$-non-amplified SK-N-AS cells and SK-N-F1 cells $(P<0.01)$, but not in $M Y C N$-amplified SK-N-DZ cells and BE(2)-M17 cells, supporting the in vivo experiment results (Supplementary Figure S2). To further investigate the underlying mechanism of poly(I:C) treatment in NB, samples of tumor tissues were taken from AS xenografts on day 17 to carry out following experiments.

To assess whether poly(I:C) affects tumor cell growth in AS xenografts, we examined the expression status of Ki-67, a marker of cell proliferation activity, in xenograft sections by IHC. Comparing with that of the control group, the xenografts with poly(I:C) treatment showed a significantly decreased Ki-67 staining $(P<0.001$, Figures $2 \mathrm{a}$ and $\mathrm{b})$. Furthermore, the xenograft sections were analyzed by IHC for phospho-growthassociated protein 43 (GAP43) expression, which is associated with cell proliferation activity in mammalian neurogenesis. ${ }^{32-34}$ Much fewer phospho-GAP43 staining cells were found in the AS xenografts with poly $(\mathrm{I}: \mathrm{C})$ treatment $(\mathrm{IRS}=0$ ) compared with the control (IRS $=8.6, P<0.001$, Figures $2 \mathrm{a}$ and c). These results indicate that AS (MYCN-non-amplified) xenografts are more sensitive towards treatment with poly(I:C) than DZ (MYCN-amplified) xenografts are.

\section{Inhibition of c-Myc and Overexpression of TLR3 are in Response to Poly(I:C) Treatment in AS Xenografts}

Our previous studies have revealed that the levels of c-Myc are associated with TLR3 expression in NB patients and TLR3induced signaling in NB cells..$^{21,26,27}$ However, the role of $\mathrm{c}-\mathrm{Myc}$ in the innate immune system is still unknown. To uncover whether c-Myc expression is influenced by TLR3mediated innate immunity, we first compared c-Myc and TLR3 expressions by IHC between the control and poly(I:C) treatment groups of AS xenografts. Poly(I:C) dramatically downregulated c-Myc protein expression with staining in $<10 \%$ tumor and an IRS of 0.25 compared with an IRS of 6.13 in the control group $(P=0.046$, Figures $3 \mathrm{a}$ and $\mathrm{b})$. On the contrary, the expressions of TLR3 and phosphorylated IRF3 (p-IRF3) by IHC were increased in AS xenografts after poly(I:C) treatment (Figures $3 \mathrm{c}-\mathrm{e})$. These results suggest that poly(I:C)-mediated innate immune signaling is able to be transducted by upregulation of TLR3 that may hamper c-Myc expression in xenograft models. a
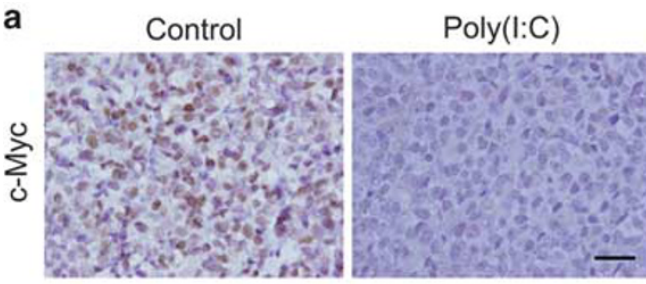

C

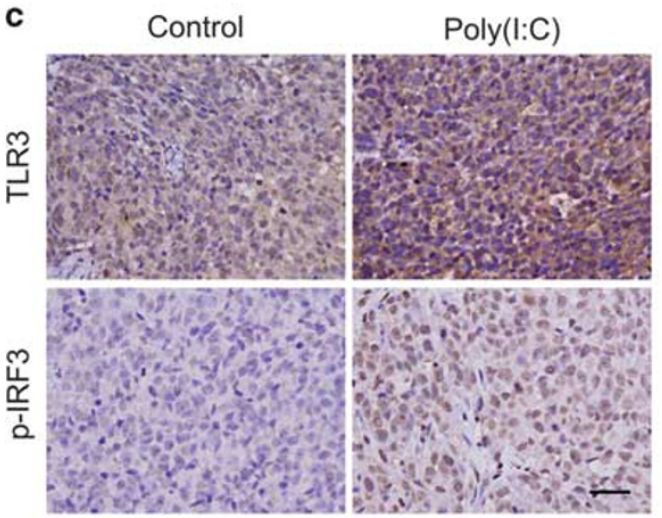

b
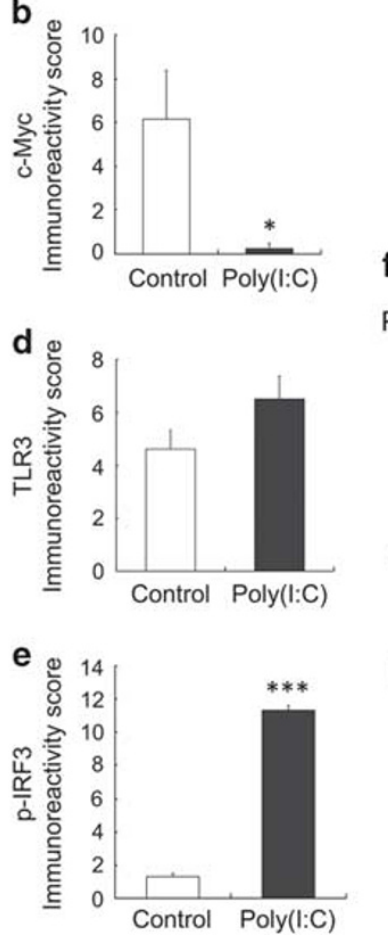

f

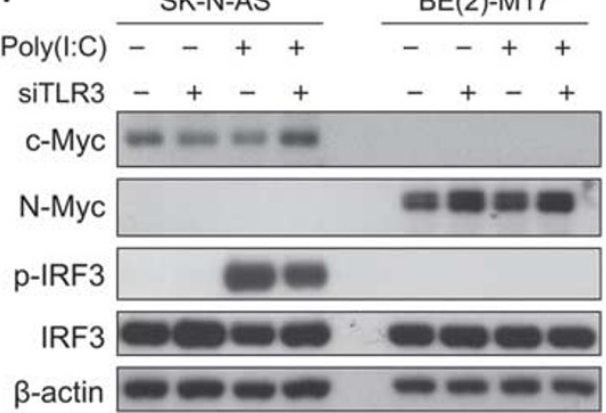

Figure 3 The expression of c-Myc, TLR3 and p-IRF3 were regulated by poly(l:C) in SK-N-AS xenografts. (a and c) Representative images of immunohistochemical staining for C-Myc, TLR3, and p-IRF3 performed on tissue sections from SK-N-AS xenografts with or without poly(l:C) treatment. (b, d and e) Quantitative analyses of c-Myc, TLR3, and p-IRF3 by immunoreactivity scores. Decreased c-Myc expression and increased TLR3 and p-IRF3 expression were found in poly(I:C)-treated SK-N-AS xenografts. Data represent mean immunoreactivity score \pm s.e.m. for each group. (f) Western blot for c-Myc, N-Myc, p-IRF3, and IRF3 protein expressions was examined in SK-N-AS and BE(2)-M17 cells with poly(I:C) treatment and silencing of TLR3. Original magnification, $\times 400$. Scale bar, $25 \mu \mathrm{m}$. ${ }^{*} P<0.05,{ }^{* * *} P<0.001$. 


\section{TLR3 Facilitates the Suppression of c-Myc Expression in Poly(l:C)-Treated AS Cells}

In accordance with the above findings, we hypothesized that alteration of protein levels of c-Myc might be regulated by TLR3 signaling to facilitate poly(I:C)-induced NB cell death. To uncover the relationship between c-Myc and TLR3 in innate immunity for treatment of NB, we treated two NB cell lines, MYCN-non-amplified SK-N-AS (AS) cells and MYCNamplified BE(2)-M17 cells, with TLR3 siRNA or poly(I:C). In both cell lines, poly(I:C) treatment did increase TLR3 mRNA expression (Supplementary Figure S3). As shown in Figure 3f, poly(I:C) can induce upregulation of p-IRF3 and downregulation of c-Myc only in AS cells, but not in BE(2)-M17 cells. Silencing of TLR3 by siRNA slightly suppressed c-Myc expression, but did not affect the expression of p-IRF3 in AS cells (Figure $3 \mathrm{f}$ and Supplementary Figure S4). Comparing with only poly(I:C) treatment, c-Myc expression was reversed while poly(I:C) and siTLR3 were simultaneously used to treat AS cells. Conversely, knockdown of TLR3 or in combination of poly(I:C) treatment did not affect the expression of c-Myc and p-IRF3 in $\mathrm{BE}(2)-\mathrm{M} 17$ cells. A slight upregulation of $\mathrm{N}-\mathrm{Myc}$ protein expression was found in $\mathrm{BE}(2)-\mathrm{M} 17$ cells with siTLR3, but no differences were shown in only poly(I:C)treated cells. These results demonstrate that poly(I:C) could trigger TLR3 signaling to hamper activation of c-Myc only in $M Y C N$-non-amplified NB cells.

\section{Poly(I:C) Significantly Increased Nuclear Factor KappaB Activation and Reactive Oxygen Species Generation}

It has been known that the activation of nuclear factor kappaB $(\mathrm{NF}-\mathrm{kB})$ is involved in TLR3-mediated signals in NB cells. ${ }^{26}$ To explore whether poly(I:C) could also regulate the activation of NF- $\mathrm{\kappa B}$ in AS xenografts, p-NF- $\mathrm{KB}$ p65 expressions between treated and untreated xenografts were analyzed by IHC. The results showed that $\mathrm{p}-\mathrm{NF}-\mathrm{\kappa B}$ p65 expression was increased in the treatment group compared with that in the control group (Figures $4 \mathrm{a}$ and b). MnSOD, also known as SOD2, facilitates mitochondrial ROS hydrogen peroxide production. Transcription of MnSOD is regulated by NF$\kappa \mathrm{B}$, and involved in TLR3-mediated NB cell death. ${ }^{26,35} \mathrm{~A}$ significantly increased MnSOD expression was found in poly (I:C)-treated AS xenografts $(P<0.001$, Figures $4 \mathrm{c}$ and d). Furthermore, ROS was visualized by 8 -hydroxy-2'-deoxyguanosine $(8-\mathrm{OHdG})$, revealing a stronger immunostaining of 8OHdG in nuclei of tumor cells in poly(I:C)-treated xenografts than in controls $(P<0.001$, Figures $4 \mathrm{c}$ and e). These results indicate that poly(I:C) facilitates NF- $\kappa B$ activation and MnSOD-mediated ROS generation.

\section{TLR3-Induced Apoptosis by Upregulating Active Caspase 3 and Cleaved Poly (ADP-ribose) Polymerase in vivo}

To further elucidate whether poly(I:C) induces apoptosis in $\mathrm{NB}$ in vivo, we used activation state-specific antibody of caspase 3 for IHC on the xenograft tissue. The expression levels of active caspase 3 were significantly elevated with poly
(I:C) treatment $(P<0.001$, Figures $5 \mathrm{a}$ and $\mathrm{b})$. Moreover, it has been known that active nuclear protein PARP-1 could be recruited to the sites of DNA breaks and involved in DNA repair, whereas PARP-1 may be inactivated due to cleavage by active caspase $3.36,37$ To verify whether the level of cleaved PARP-1 was changed in response to poly(I:C) treatment, we evaluated cleaved PARP-1 expression in tissue sections of AS xenografts with or without poly(I:C) treatment. Cleaved PARP-1 was significantly higher in the treatment group (IRS = 5.71) than in the control group (IRS $=1.96, P<0.05$, Figures $5 \mathrm{a}$ and $\mathrm{c}$ ). These results demonstrate that TLR3mediated innate immunity may induce apoptosis through DNA damage in NB xenografts.

\section{DISCUSSION}

Innate immunity is contributed to protect host against pathogens. TLR3 can recognize viral double-stranded RNA to initiate innate immune responses against infection. ${ }^{22}$ Our previous studies have revealed that TLR3 has a key role in triggering innate immune responses to induce tumor cell death in NB, and its expression is associated with nonamplified MYCN in NB by clinicopathologic and biological study. ${ }^{20}$ In addition, our previous in vitro studies revealed that poly(I:C) can induce apoptotic signals in $M Y C N$-nonamplified human NB cell lines, such as SK-N-AS and SK$\mathrm{N}$-FI, but not in $M Y C N$-amplified human NB cell lines. ${ }^{21,26}$

Here we study the effects of TLR3-agonist poly(I:C) treatment in NB xenografts. Our data show that poly(I:C) significantly suppressed tumor growth in $M Y C N$-non-amplified AS xenografts, but not in $M Y C N$-amplified DZ xenografts. Activation of GAP43 has been known to mediate axonal proliferation and regeneration, proliferating neuroepithelium as well as horizontal cell divisions during mammalian neurogenesis. ${ }^{32-34}$ In the present study, reduced expression of proliferation markers, Ki-67 and p-GAP43, was found in poly(I:C)-treated AS xenografts (Figure 2). Moreover, no apparent pathological changes of liver and kidney were found in mice after poly(I:C) treatment (Supplementary Figure S5) in histopathology. Although higher levels of blood urea nitrogen (BUN; $P=0.044$ ) and alanine aminotransferase (ALT; $P=0.043$ ) were found in treatment group compared with control group (Supplementary Table S1), levels of the liver enzymes and the kidney enzymes were within the normal range. ${ }^{38}$ These results indicate that $\mathrm{c}-\mathrm{Myc}$ protein expression might be susceptible to poly(I:C)-induced NB cell death.

In the present study, downregulation of $\mathrm{c}-\mathrm{Myc}$ and upregulation of TLR3 were found in poly(I:C)-treated AS xenografts. However, the relationship between $\mathrm{c}-\mathrm{Myc}$ and TLR3 in treatment of poly(I:C) for cancer remains inconsistent. It has been reported that $\mathrm{c}-\mathrm{Myc}$ oncoprotein has a critical role in maintaining the balance between proliferation and apoptosis in tumor progression in vivo. ${ }^{39}$ Positive TLR3 expression was considered a favorable prognostic factor whereas positive c-Myc protein expression is a poor prognostic factor by clinical research of NB. ${ }^{8,20}$ However, 
a

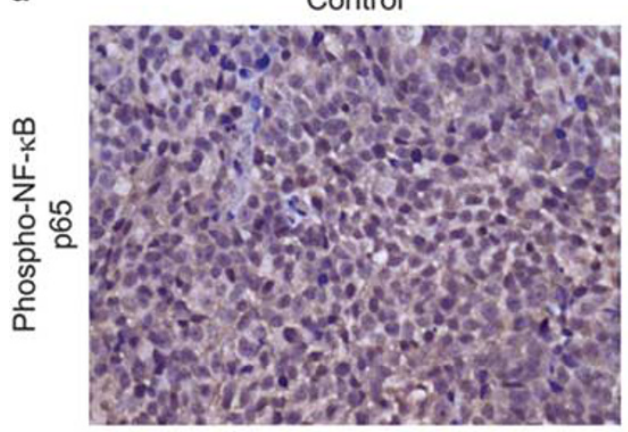

C
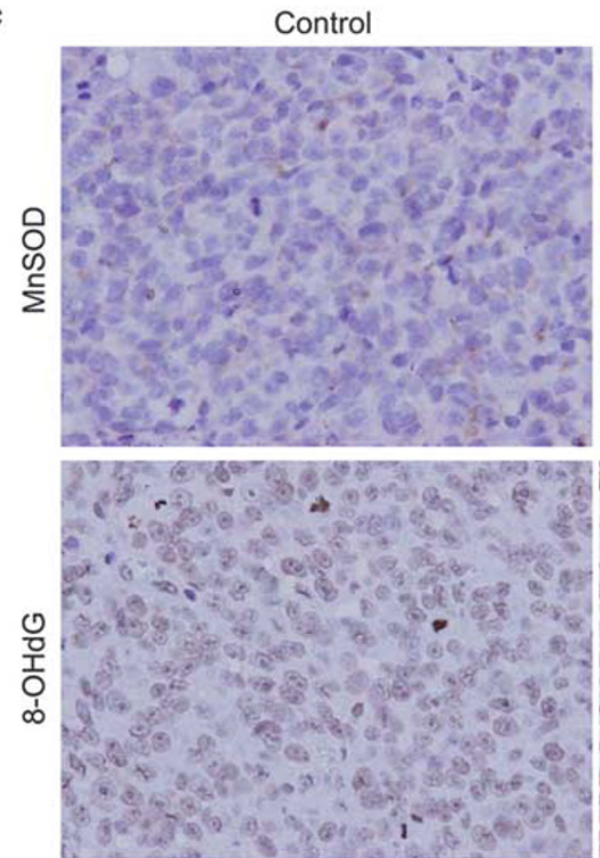

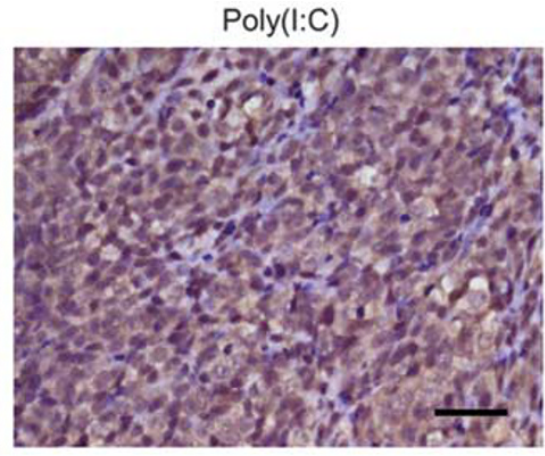

Poly(I:C)
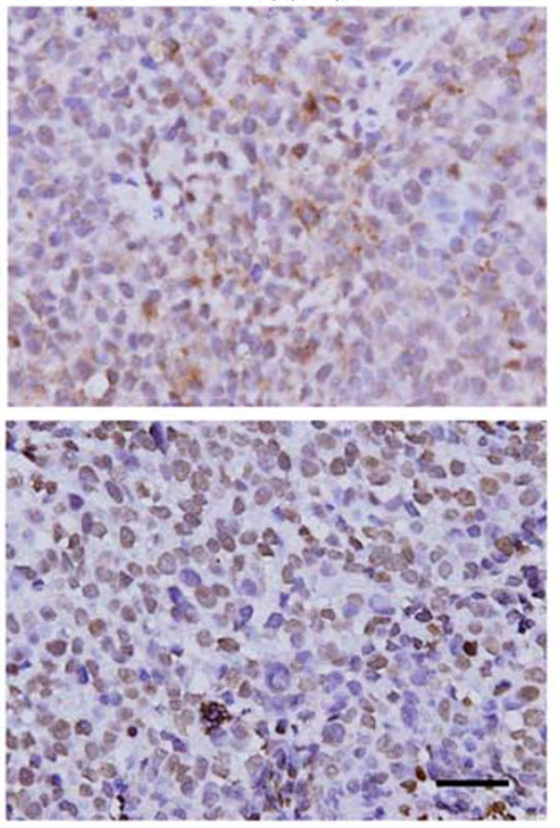

b

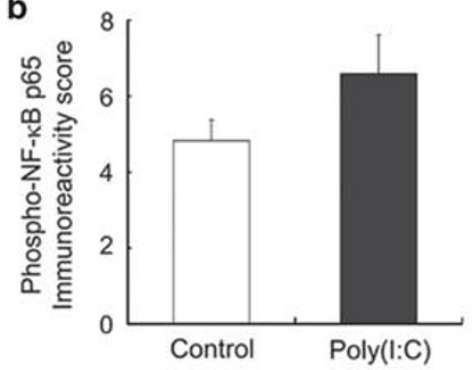

d
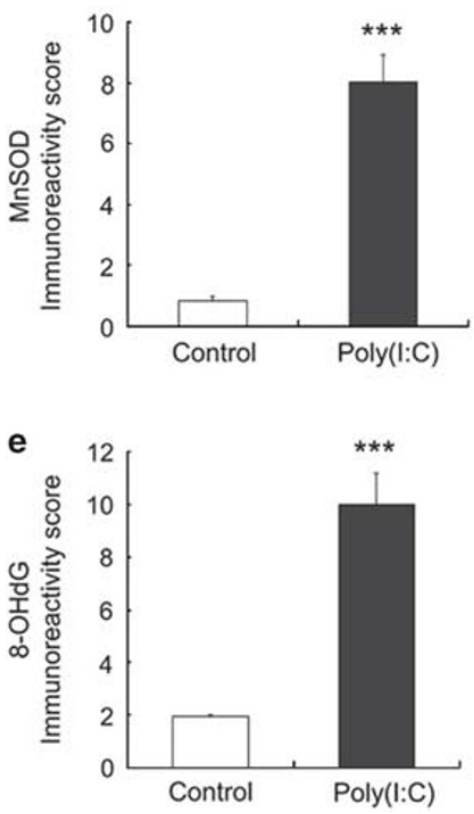

Figure 4 ROS generation induced by poly(I:C) treatment in SK-N-AS xenografts. (a and c) Representative images of immunohistochemical staining for p-NF-kB p65, MnSOD, and 8-OHdG performed on tissue sections from SK-N-AS xenografts with or without poly(l:C) treatment. (b, d and e) Quantitative analyses of p-NF-kB p65, MnSOD, and 8-OHdG by immunoreactivity scores. p-NF-kB p65, MnSOD, and 8-OHdG were upregulated in poly(I:C)-treated AS xenografts compared with the control. Data represent mean immunoreactivity score \pm s.e.m. for each group. Original magnification, $\times 400$. Scale bar, $25 \mu \mathrm{m} .{ }^{* * *} P<0.001$.

elevated TLR3 expression in breast cancer and overexpression of c-Myc in HER2-positive breast cancer were respectively determined as a poor prognostic factor, ${ }^{40,41}$ and TLR3 treatment was associated with overexpression of c-Myc to facilitate stem cells potential. ${ }^{40}$ In addition, overexpression of TLR3 and c-Myc was reported to be induced by poly(I:C) treatment, which led to cell proliferation in head and neck cancer. ${ }^{42}$ These reports indicate that the roles of TLR3 and c-Myc in innate immune system and their responses to poly(I:C) treatment might be type-specific for cancer. Nevertheless, our in vitro results demonstrate that TLR3 mRNA is upregulated by poly(I:C) treatment in AS cells and BE(2)-M17 cells (Supplementary Figure S3), but IRF3 is only activated in poly (I:C)-treated AS cells but not in BE(2)-M17 cells (Figure 3f). Moreover, treatment with TLR3 siRNA and poly(I:C) in AS cells could attenuate the downregulation of c-Myc induced by poly(I:C) alone treatment, suggesting that poly(I:C) suppresses c-Myc expression in TLR3-mediated innate immunity.

Interestingly, we found that knockdown of TLR3 in AS cells can decrease c-Myc protein expression similar to poly(I:C) treatment, but which could not completely suppress the expression of poly(I:C)-induced p-IRF3. Several studies have demonstrated that small interfering RNAs (siRNAs) may be recognized by several TLRs (TLR3, TLR7, and TLR8) to induce unanticipated vascular or immune effects. ${ }^{43,44}$ Our previous studies also found that knockdown of other cytosolic RNA receptors, MDA5 and RIG-1, can decrease poly(I:C)induced p-IRF3 expression in NB cells. ${ }^{27}$ These studies clearly indicate that other TLRs signaling and cytosolic RNA receptors might be involved in the regulation of c-Myc and p-IRF3 expression while AS cells were treated with siTLR3. 
a
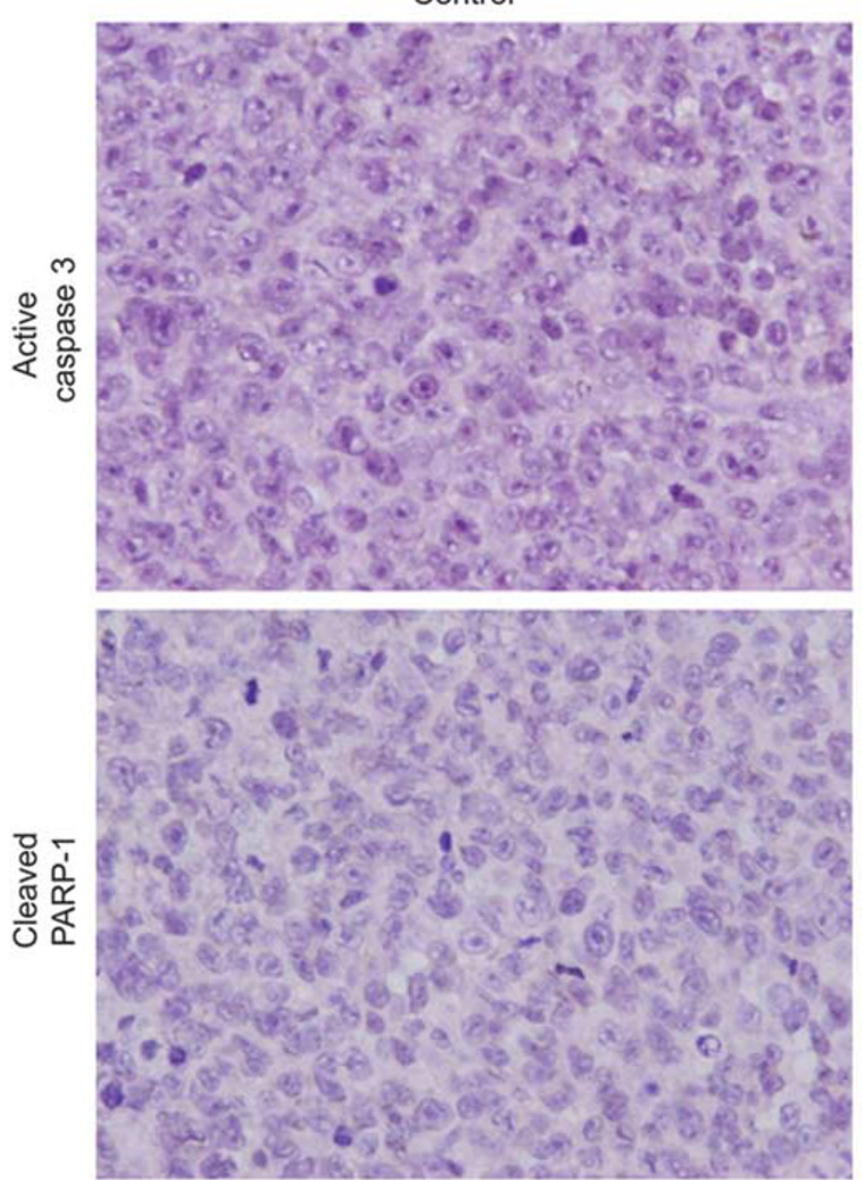

b

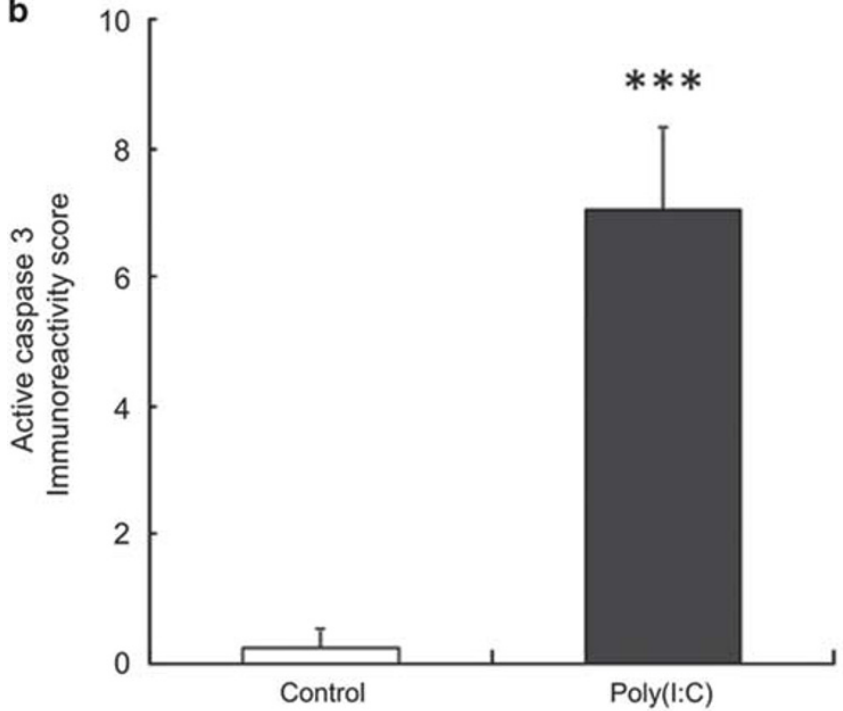

Poly(I:C)
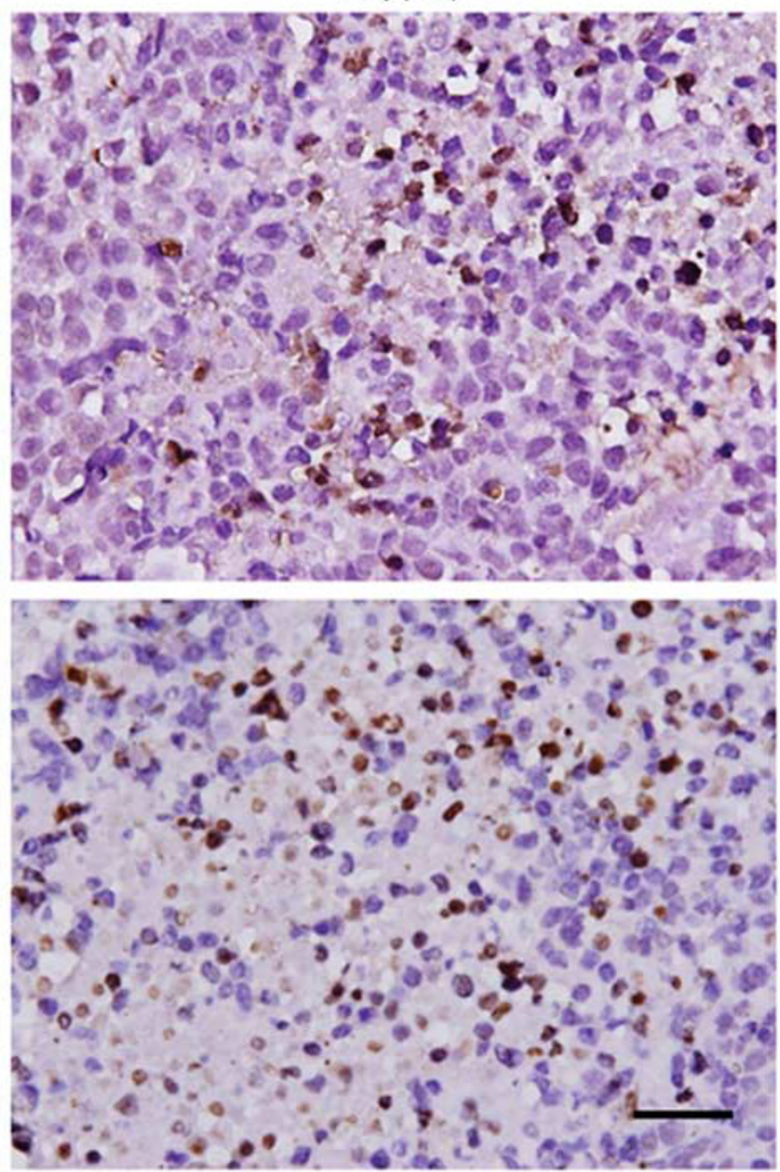

C

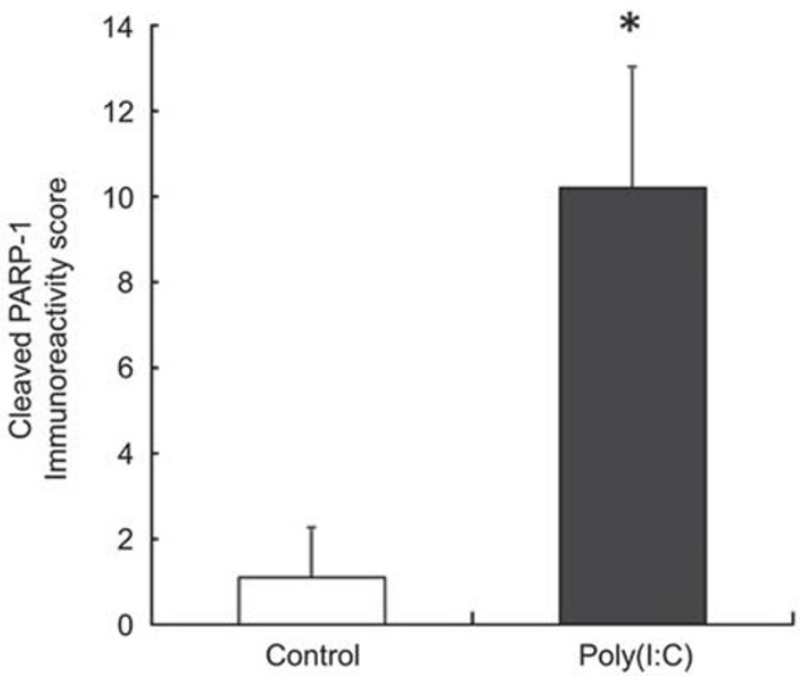

Figure 5 Activation of caspase 3 and cleavage of PARP-1 shown as evidence of apoptosis induced by poly(l:C) treatment in SK-N-AS xenografts. (a) Representative images of immunohistochemical staining for active caspase 3 and cleaved PARP-1 performed on tissue sections from SK-N-AS xenografts with or without poly(l:C) treatment. (b and $\mathbf{c})$ Quantitative analyses of active caspase 3 and cleaved PARP-1 by immunoreactivity scores. Data represent mean immunoreactivity score \pm s.e.m. for each group. Original magnification, $\times 400$. Scale bar, $25 \mu \mathrm{m}$. ${ }^{*} P<0.05,{ }^{* * *} P<0.001$. 


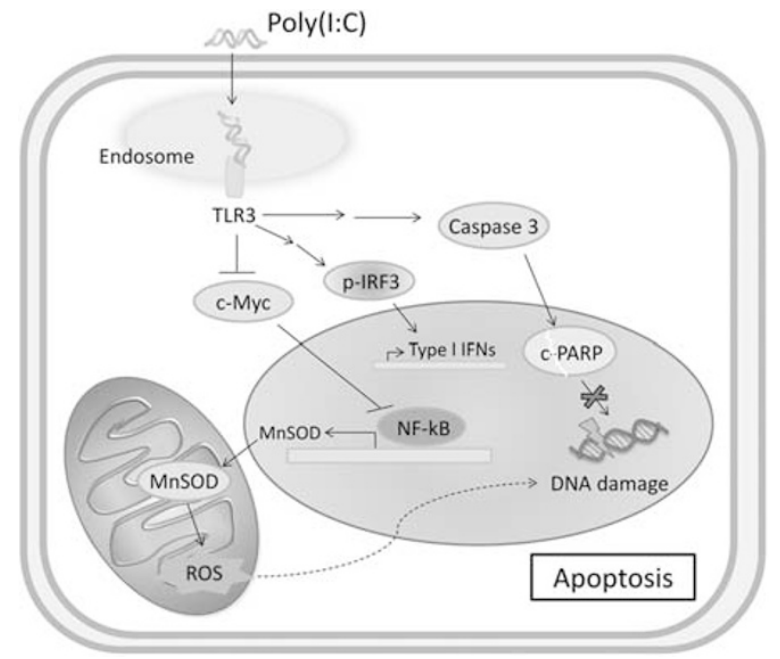

Figure 6 Illustration for the relationship among TLR3-agonist poly(l:C), c-Myc, NF-kB, and ROS in innate immune responses. Poly $(\mathrm{l}: \mathrm{C})$ treatment may trigger TLR3 signaling to initiate innate immunity followed by activation of IRF3 and type I IFNs. Subsequently, c-Myc is downregulated by poly $(\mathrm{l}: \mathrm{C})$, resulting in activation of $\mathrm{NF}-\mathrm{KB}$, followed by overexpression of MnSOD, a NF-KB transcriptional target gene, to facilitate ROS generation. TLR3 signaling also activates caspase 3 to cleave PARP-1 to impair DNA repair. Finally, apoptosis and growth arrest are induced by TLR3-mediated innate immune responses in MYCN-non-amplified NB xenografts.

Even if we have selected two different TLR3 siRNAs to avoid off-target effects, unanticipated innate immune signaling still could not be completely excluded (Figure $3 \mathrm{f}$ and Supplementary Figure S4).

It has been known that functional diversity of TLR3 in tumors can mediate the fates of cancer cells. Through triggering NF- $\mathrm{KB}$ activation, TLR3 signal transduction may facilitate multiple myeloma cell survival. ${ }^{45}$ It is also reported that TLR3 can promote inflammatory via mTOR to activate NF- $\mathrm{\kappa B}$ in human oral keratinocytes. ${ }^{46}$ In innate immunity, TLR3 triggers the activation of NF- $\mathrm{KB}$ and IRF3, generates type I interferons (IFNs) and promotes dendritic cell maturation. ${ }^{47,48}$ Viral infection activates transactivation potential of IRF3 by regulating phosphorylation of site 1 (Ser385 and Ser386) and site 2 (Ser396, Ser398, Ser402, Thr404, and Ser405). ${ }^{4-51}$ Our data suggest that poly(I:C) treatment triggers the activation of NF- $\mathrm{KB}$ and IRF3 in AS xenografts. These results indicate that poly(I:C) could induce innate immunity by regulating activation of NF- $\mathrm{KB}$ in AS xenografts.

Our data show that poly(I:C) may upregulate MnSOD and 8-OHdG expression in AS xenografts. 8-OHdG acts as a pivotal marker to detect oxidative stress and to indicate oxidative DNA damage. ${ }^{52}$ Deregulation of c-Myc could cause growth arrest due to inhibition of c-Myc transcriptional target genes, that act as positive regulators of cell cycle progression, survival, protein synthesis, and energy-consuming processes. ${ }^{53,54}$ It has been known that $\mathrm{c}-\mathrm{Myc}$ downregulating NF- $\mathrm{KB}$ is involved in immune escape in cancer pathogenesis. ${ }^{55,56}$ Phosphorylated $\mathrm{NF}-\mathrm{\kappa B}$ can promote MnSOD transcriptional expression to enhance ROS hydrogen peroxide generation during nutritional deprivation. ${ }^{56}$ c-Myc inactivation and DNA lesion, both of which may trigger cell cycle arrest, cause subsequent growth arrest or apoptosis. ${ }^{17,57,58}$ Taken together, poly(I:C) may induce tumor death through TLR3/c-Myc/NF-kB pathway.

Furthermore, cleavage of PARP-1 is a signature of neurodegeneration, and involved in neuronal death in the nervous system. ${ }^{59,60}$ PARP-1, an chromatin-associated enzyme, can be induced by DNA breakage to orchestrate DNA base excision repair and in response to apoptosis occurring. ${ }^{61}$ Activated caspase 3 cleaves and inactivates PARP-1 in late apoptotic cells. In the present study, after poly(I:C) treatment, activated caspase 3 and cleaved PARP-1 were significantly upregulated, resulting in introduction of apoptosis in AS xenografts.

In summary, we demonstrated that TLR3-mediated innate immune responses can significantly eliminate tumor growth in $M Y C N$-non-amplified AS xenografts, but not in $M Y C N$ amplified DZ xenografts. Suppression of tumor cell proliferation by poly(I:C) in AS xenografts was determined by the downregulation of $\mathrm{Ki}-67$ and p-GAP43 immunostaining. Significant downregulation of c-Myc, and upregulation of TLR3, p-NF-kB p65, p-IRF3, MnSOD, 8-OHdG, active caspase 3 as well as cleaved PARP-1 were induced by poly(I: C) treatment in AS xenografts. Knockdown of TLR3 blocked the inhibition of c-Myc protein expression regulated by poly (I:C) in AS cells. As shown in Figure 6, we propose that TLR3 suppresses c-Myc to upregulate the activation of NF- $\mathrm{kB}$, which induces MnSOD-mediated ROS generation, in poly(I: C)-treated AS xenografts. Subsequently, DNA damage could be induced by caspase 3-catalyzed PARP cleavage during apoptosis. In this study, we suggest that high c-Myc protein expression may increase tumor cell sensitivity to poly(I:C) treatment in NB xenografts, which is involved in TLR3mediated tumor growth arrest and apoptosis. Thus, these results provide a new insight about the role of $\mathrm{c}-\mathrm{Myc}$ protein in innate immune system for the treatment of NB, so that future clinical research and drug development can improve immunotherapy strategies.

Supplementary Information accompanies the paper on the Laboratory Investigation website (http://www.nature.com/ labinvest).

Supplementary Information accompanies the paper on the Laboratory Investigation website (http://www.laboratoryinvestigation.org)

\section{ACKNOWLEDGMENTS}

This study was supported by grants from the Chang Gung Memorial Hospital CMRPG8C0393, Taiwan. We thank Chang Gung Medical Foundation Kaohsiung Chang Gung Memorial Hospital Tissue Bank (CLRPG8B0033 and CLRPG8E0161) for excellent technical support.

\section{DISCLOSURE/CONFLICT OF INTEREST}

The authors declare no conflict of interest. 
1. Maris $\mathrm{JM}$, Hogarty $\mathrm{MD}$, Bagatell $\mathrm{R}$ et al. Neuroblastoma. Lancet 2007;369:2106-2120.

2. Mueller S, Matthay KK. Neuroblastoma: biology and staging. Curr Oncol Rep 2009;11:431-438.

3. Franks $L M$, Bollen A, Seeger RC et al. Neuroblastoma in adults and adolescents: an indolent course with poor survival. Cancer 1997;79: 2028-2035.

4. Brodeur GM. Neuroblastoma: biological insights into a clinical enigma. Nat Rev Cancer 2003;3:203-216.

5. Cohn $S L$, Pearson $A D$, London $W B$ et al. The International Neuroblastoma Risk Group (INRG) classification system: an INRG Task Force report. J Clin Oncol 2009;27:289-297.

6. Baker DL, Schmidt ML, Cohn SL et al. Outcome after reduced chemotherapy for intermediate-risk neuroblastoma. N Engl J Med 2010:363:1313-1323.

7. Strother DR, London $\mathrm{WB}, \mathrm{Schmidt} \mathrm{ML}$ et al. Outcome after surgery alone or with restricted use of chemotherapy for patients with low-risk neuroblastoma: results of Children's Oncology Group study P9641. J Clin Oncol 2012:30:1842-1848.

8. Wang LL, Suganuma $\mathrm{R}$, Ikegaki $\mathrm{N}$ et al. Neuroblastoma of undifferentiated subtype, prognostic significance of prominent nucleolar formation, and MYC/MYCN protein expression: a report from the Children's Oncology Group. Cancer 2013;119:3718-3726.

9. Schneiderman J, London WB, Brodeur GM et al. Clinical significance of MYCN amplification and ploidy in favorable-stage neuroblastoma: a report from the Children's Oncology Group. J Clin Oncol 2008;26: 913-918.

10. Cohn SL, Look AT, Joshi VV et al. Lack of correlation of N-myc gene amplification with prognosis in localized neuroblastoma: a Pediatric Oncology Group study. Cancer Res 1995:55:721-726.

11. Perez CA, Matthay KK, Atkinson JB et al. Biologic variables in the outcome of stages I and II neuroblastoma treated with surgery as primary therapy: a children's cancer group study. J Clin Oncol 2000;18: $18-26$.

12. Westermann F, Muth D, Benner A et al. Distinct transcriptional MYCN/ c-MYC activities are associated with spontaneous regression or malignant progression in neuroblastomas. Genome Biol 2008;9:R150.

13. Valentijn LJ, Koster J, Haneveld $\mathrm{F}$ et al. Functional MYCN signature predicts outcome of neuroblastoma irrespective of MYCN amplification. Proc Natl Acad Sci USA 2012;109:19190-19195.

14. Ikegaki N, Shimada H, Fox $A M$ et al. Transient treatment with epigenetic modifiers yields stable neuroblastoma stem cells resembling aggressive large-cell neuroblastomas. Proc Natl Acad Sci USA 2013;110:6097-6102.

15. Dang CV. MYC on the path to cancer. Cell 2012;149:22-35.

16. Hoffman B, Liebermann DA. Apoptotic signaling by c-MYC. Oncogene 2008;27:6462-6472

17. Felsher DW. MYC inactivation elicits oncogene addiction through both tumor cell-intrinsic and host-dependent mechanisms. Genes Cancer 2010;1:597-604.

18. Yu AL, Gilman AL, Ozkaynak MF et al. Anti-GD2 antibody with GM-CSF, interleukin-2, and isotretinoin for neuroblastoma. N Engl J Med 2010:363:1324-1334.

19. Seeger RC. Immunology and immunotherapy of neuroblastoma. Semin Cancer Biol 2011:21:229-237.

20. Hsu WM, Huang CC, Wu PY et al. Toll-like receptor 3 expression inhibits cell invasion and migration and predicts a favorable prognosis in neuroblastoma. Cancer Lett 2013;336:338-346.

21. Chuang $\mathrm{JH}$, Chuang $\mathrm{HC}$, Huang CC et al. Differential toll-like receptor 3 (TLR3) expression and apoptotic response to TLR3 agonist in human neuroblastoma cells. J Biomed Sci 2011;18:65.

22. Sen GC, Sarkar SN. Transcriptional signaling by double-stranded RNA: role of TLR3. Cytokine Growth Factor Rev 2005;16:1-14.

23. Matsumoto $M$, Seya T. TLR3: interferon induction by double-stranded RNA including poly(l:C). Adv Drug Deliv Rev 2008;60:805-812.

24. Ammi $R$, De Waele J, Willemen $Y$ et al. Poly $(I: C)$ as cancer vaccine adjuvant: knocking on the door of medical breakthroughs. Pharmacol Ther 2015;146:120-131.

25. Harashima $\mathrm{N}$, Minami $\mathrm{T}$, Uemura $\mathrm{H}$ et al. Transfection of poly(l:C) can induce reactive oxygen species-triggered apoptosis and interferonbeta-mediated growth arrest in human renal cell carcinoma cells via innate adjuvant receptors and the 2-5 A system. Mol Cancer 2014; 13:217.
26. Chuang $\mathrm{JH}$, Lin TK, Tai $\mathrm{MH}$ et al. Preferential involvement of mitochondria in Toll-like receptor 3 agonist-induced neuroblastoma cell apoptosis, but not in inhibition of cell growth. Apoptosis 2012;17: 335-348.

27. Hsu WM, Huang CC, Lee HY et al. MDA5 complements TLR3 in suppression of neuroblastoma. Oncotarget 2015;6:24935-24946.

28. Thompson J, Guichard SM, Cheshire PJ et al. Development, characterization and therapy of a disseminated model of childhood neuroblastoma in SCID mice. Cancer Chemother Pharmacol 2001;47: 211-221.

29. Amoh $Y$, Nagakura $C$, Maitra A et al. Dual-color imaging of nascent angiogenesis and its inhibition in liver metastases of pancreatic cancer. Anticancer Res 2006;26:3237-3242.

30. Bao YX, Cao Q, Yang $Y$ et al. Expression and prognostic significance of golgiglycoprotein73 (GP73) with epithelial-mesenchymal transition (EMT) related molecules in hepatocellular carcinoma (HCC). Diagn Pathol 2013;8:197.

31. Sinicrope FA, Ruan SB, Cleary KR et al. bcl-2 and p53 oncoprotein expression during colorectal tumorigenesis. Cancer Res 1995;55: 237-241.

32. Martzen MR, Nagy A, Coleman PD et al. Altered phosphorylation of growth-associated protein B50/GAP-43 in Alzheimer disease with high neurofibrillary tangle density. Proc Natl Acad Sci USA 1993;90: 11187-11191.

33. Shen $Y$, Mishra R, Mani $S$ et al. Both cell-autonomous and cell nonautonomous functions of GAP-43 are required for normal patterning of the cerebellum in vivo. Cerebellum 2008;7:451-466.

34. Stricker $\mathrm{SH}$, Meiri K, Gotz M. P-GAP-43 is enriched in horizontal cell divisions throughout rat cortical development. Cereb Cortex 2006;16 (Suppl 1):i121-i131.

35. Baud V, Karin M. Is NF-kappaB a good target for cancer therapy? Hopes and pitfalls. Nat Rev Drug Discov 2009;8:33-40.

36. Kaufmann SH, Desnoyers S, Ottaviano $\mathrm{Y}$ et al. Specific proteolytic cleavage of poly(ADP-ribose) polymerase: an early marker of chemotherapy-induced apoptosis. Cancer Res 1993:53:3976-3985.

37. Tewari M, Quan LT, O'Rourke $\mathrm{K}$ et al. Yama/CPP32 beta, a mammalian homolog of CED-3, is a CrmA-inhibitable protease that cleaves the death substrate poly(ADP-ribose) polymerase. Cell 1995;81:801-809.

38. RAR (Research Animal Resources) University of Minnesota, United States., $2011 \mathrm{http}: / / \mathrm{www}$. ahc.umn.edu/rar/refvalues.html.

39. Pelengaris $\mathrm{S}$, Rudolph $\mathrm{B}$, Littlewood $\mathrm{T}$. Action of Myc in vivo - proliferation and apoptosis. Curr Opin Genet Dev 2000;10:100-105.

40. Jia D, Yang W, Li L et al. beta-Catenin and NF-kappaB co-activation triggered by TLR3 stimulation facilitates stem cell-like phenotypes in breast cancer. Cell Death Differ 2015;22:298-310.

41. Nair R, Roden DL, Teo WS et al. c-Myc and Her2 cooperate to drive a stem-like phenotype with poor prognosis in breast cancer. Oncogene 2014:33:3992-4002.

42. Pries R, Hogrefe L, Xie L et al. Induction of c-Myc-dependent cell proliferation through toll-like receptor 3 in head and neck cancer. Int J Mol Med 2008;21:209-215.

43. Kleinman ME, Yamada $K$, Takeda $A$ et al. Sequence- and targetindependent angiogenesis suppression by siRNA via TLR3. Nature 2008;452:591-597.

44. Jackson AL, Linsley PS. Recognizing and avoiding siRNA off-target effects for target identification and therapeutic application. Nat Rev Drug Discov 2010;9:57-67.

45. Chiron D, Pellat-Deceunynck $C$, Amiot $M$ et al. TLR3 ligand induces $\mathrm{NF}-\{k a p p a\} \mathrm{B}$ activation and various fates of multiple myeloma cells depending on IFN-\{alpha\} production. J Immunol 2009;182:4471-4478.

46. Zhao J, Benakanakere MR, Hosur KB et al. Mammalian target of rapamycin (mTOR) regulates TLR3 induced cytokines in human oral keratinocytes. Mol Immunol 2010;48:294-304.

47. Alexopoulou L, Holt AC, Medzhitov R et al. Recognition of doublestranded RNA and activation of NF-kappaB by Toll-like receptor 3 . Nature 2001:413:732-738.

48. Doyle S, Vaidya S, O'Connell R et al. IRF3 mediates a TLR3/TLR4-specific antiviral gene program. Immunity 2002;17:251-263.

49. Panne D, McWhirter SM, Maniatis T et al. Interferon regulatory factor 3 is regulated by a dual phosphorylation-dependent switch. J Biol Chem 2007;282:22816-22822.

50. Lin R, Heylbroeck C, Pitha PM et al. Virus-dependent phosphorylation of the IRF-3 transcription factor regulates nuclear translocation, 
transactivation potential, and proteasome-mediated degradation. Mol Cell Biol 1998;18:2986-2996.

51. Yoneyama $M$, Suhara $W$, Fukuhara $Y$ et al. Direct triggering of the type I interferon system by virus infection: activation of a transcription factor complex containing IRF-3 and CBP/p300. EMBO J 1998;17: 1087-1095.

52. Shigenaga MK, Gimeno CJ, Ames BN. Urinary 8-hydroxy-2'deoxyguanosine as a biological marker of in vivo oxidative DNA damage. Proc Natl Acad Sci USA 1989;86:9697-9701.

53. Dang CV. c-Myc target genes involved in cell growth, apoptosis, and metabolism. Mol Cell Biol 1999;19:1-11.

54. Schmidt EV. The role of c-myc in cellular growth control. Oncogene 1999;18:2988-2996.

55. Schlee M, Holzel M, Bernard S et al. C-myc activation impairs the NF-kappaB and the interferon response: implications for the pathogenesis of Burkitt's lymphoma. Int J Cancer 2007;120:1387-1395.
56. Tanaka H, Matsumura I, Ezoe $\mathrm{S}$ et al. E2F1 and c-Myc potentiate apoptosis through inhibition of NF-kappaB activity that facilitates MnSOD-mediated ROS elimination. Mol Cell 2002;9:1017-1029.

57. Pellegata NS, Antoniono RJ, Redpath JL et al. DNA damage and p53mediated cell cycle arrest: a reevaluation. Proc Natl Acad Sci USA 1996;93:15209-15214.

58. Ho JS, Ma W, Mao DY et al. p53-Dependent transcriptional repression of c-myc is required for $\mathrm{G} 1$ cell cycle arrest. Mol Cell Biol 2005;25:7423-7431.

59. Chaitanya GV, Steven AJ, Babu PP. PARP-1 cleavage fragments: signatures of cell-death proteases in neurodegeneration. Cell Commun Signal 2010;8:31.

60. Koh DW, Dawson TM, Dawson VL. Poly(ADP-ribosyl)ation regulation of life and death in the nervous system. Cell Mol Life Sci 2005;62:760-768.

61. Lazebnik YA, Kaufmann SH, Desnoyers $S$ et al. Cleavage of poly(ADPribose) polymerase by a proteinase with properties like ICE. Nature 1994;371:346-347. 